\title{
Rapid vascularization of starch-poly(caprolactone) in vivo by outgrowth endothelial cells in co-culture with primary osteoblasts
}

\author{
Shahram Ghanaati ${ }^{1,2 \# *}$, Sabine Fuchs ${ }^{1 \#}$, Matthew J. Webber ${ }^{3}$, Carina Orth ${ }^{1}$, Mike Barbeck ${ }^{1}$, \\ Manuela E. Gomes ${ }^{4,5}$, Rui L. Reis ${ }^{4,5}$ and C. James Kirkpatrick ${ }^{1}$ \\ ${ }^{1}$ REPAIR-lab, Institute of Pathology, University Medical Center of the Johannes Gutenberg University, \& European Institute of Excellence \\ on Tissue Engineering and Regenerative Medicine, Langenbeckstrasse 1, D-55101 Mainz, Germany \\ ${ }^{2}$ Clinic for Maxillofacial and Plastic Surgery, Johann Wolfgang Goethe University, Frankfurt/Main, Germany \\ ${ }^{3}$ Department of Biomedical Engineering, Northwestern University, Evanston, IL 60208, USA \\ ${ }^{4} 3 B$ 's Research Group - Biomaterials, Biodegradables and Biomimetics, Dept. of Polymer Eng., Univ. of Minho, Headquarters of the \\ European Institute of Excellence on Tissue Engineering and Regenerative Medicine, AvePark, 4806-909 Taipas, Guimarães, Portugal \\ ${ }^{5}$ IBB-Institute for Biotechnology and Bioengineering, PT Government Associated Laboratory, Guimarães, Portugal
}

\begin{abstract}
The successful integration of in vitro-generated tissues is dependent on adequate vascularization in vivo. Human outgrowth endothelial cells (OECs) isolated from the mononuclear cell fraction of peripheral blood represent a potent population of circulating endothelial progenitors that could provide a cell source for rapid anastomosis and scaffold vascularization. Our previous work with these cells in co-culture with primary human osteoblasts has demonstrated their potential to form perfused vascular structures within a starch-poly(caprolactone) biomaterial in vivo. In the present study, we demonstrate the ability of OECs to form perfused vascular structures as early as $48 \mathrm{~h}$ following subcutaneous implantation of the biomaterial in vivo. The number of OECderived vessels increased throughout the study, an effect that was independent of the OEC donor. This finding of rapid and thorough OEC-mediated scaffold vascularization demonstrates the great potential for OEC-based strategies to promote vascularization in tissue engineering. OECs have the potential to contribute to host-derived scaffold vascularization, and formed vascular structures at a similar density as those arising from the host. Additionally, immunohistochemical evidence demonstrated the close interaction between OECs and the co-cultured osteoblasts. In addition to the known paracrine activity osteoblasts have in modulating angiogenesis of co-cultured OECs, we demonstrate the potential of osteoblasts to provide additional structural support for OEC-derived vessels, perhaps acting in a pericyte-like role. Copyright $\odot 2010$ John Wiley \& Sons, Ltd.
\end{abstract}

Received 27 July 2010; Accepted 2 September 2010

Keywords outgrowth endothelial cell; osteoblast; co-culture; angiogenesis; in vivo; pericyte

\section{Introduction}

Appropriate in vivo vascularization is paramount to the therapeutic success of in vitro-engineered tissue constructs. Outgrowth endothelial cells (OECs) represent

\footnotetext{
*Correspondence to: Shahram Ghanaati, Institute of Pathology, Langenbeckstrasse 1, Johannes Gutenberg University, 55101 Mainz, Germany. E-mail: ghanaati@uni-mainz.de

\# These authors contributed equally to this study.
}

a potentially potent population of endothelial progenitor cells that can be isolated in cultures of mononuclear cells from human peripheral blood and can be used in complex tissue engineering strategies to enhance scaffold vascularization (Fuchs et al., 2006a, 2006b, 2009a; Au et al., 2008; Melero-Martin et al., 2007; Silva et al., 2008). The ability of OECs and other endothelial cells to form vessel-like structures has been demonstrated by our group in vitro in mono- and co-culture methods in both two- and three-dimensional (2D and 3D) cultures 
(Fuchs et al., 2006a; Unger et al., 2004, 2005; Fuchs et al., 2007, 2009b. A recent study by our group found that, in co-culture with human primary osteoblasts (pOB) on starch-poly(caprolactone) fibre meshes (SPCL), OECs significantly contributed to in vivo scaffold vascularization by forming fully perfused vessels following implantation (Fuchs et al., 2009c). The contribution of the pOB component of the co-culture is crucial, as OECs seeded alone on SPCL did not demonstrate the same potency to form vascular structures in vivo in this study. Similar observations have been found for primary endothelial cells in co-culture with osteoblasts in vivo (Unger et al., 2007,2010 ). In these previous studies, we have suggested that the pOBs play a role in endothelial cell-derived vessel formation through the production of extracellular matrix and the secretion of pro-angiogenic signalling molecules, resulting in vessel-like formation in vitro (Fuchs et al., 2007; Santos et al., 2009). Accordingly, osteoblasts have been viewed as a natural source for potent signalling molecules that modulate the angiogenic behaviour of the co-cultured endothelial cells, suggesting the existence of a natural synergy between osteoblasts and endothelial cells. This phenomenon of enhanced vessel formation arising from precultured endothelial cells when in co-culture with osteoblasts could be used as a strategy for complex tissue engineering, where scaffold vascularization and rapid integration are essential for survival of the cellseeded construct. The use of osteoblasts in the co-culture could be especially suited for tissue engineering of bone, where the osteoblasts could contribute to the functional vascularization that is required for bone regeneration, while also perhaps providing bone-specific functionality.

In this study, we evaluated the time course necessary for in vitro-generated OEC-pOB co-cultures to form functional vessels anastomosed with host vasculature in vivo. Our previous reports on this phenomenon used only endpoint identification of OEC-derived vascular structures and indicated a contribution to this process when OECs were co-cultured with pOBs (Fuchs et al., 2009c)[11]. In the present study we performed a dynamic immunohistological study at multiple time points to determine when in vitro-generated OEC-pOB constructs first demonstrate anastomosis with host vasculature in vivo to form perfused vascular structures ('inosculation') within the construct. We also use immunohistological staining to determine whether the OEC and pOB cells interact following transplantation of the construct in vivo, with a view to elucidating the role pOBs play in OEC-mediated scaffold vascularization by determining whether osteoblasts structurally contribute to vascularization in addition to their previously demonstrated paracrine contribution.

\section{Materials and methods}

\subsection{Isolation of human OECs}

Outgrowth endothelial cells (OECs) were isolated according to previously established methods (Fuchs et al., 2006a, 2006b, 2007, 2009c). In brief, mononuclear cells were isolated from human peripheral blood buffy coats by Ficoll (Sigma-Aldrich)-gradient centrifugation and cultured in endothelial cell growth medium-2 (EGM-2; Lonza) complete with $5 \%$ fetal calf serum (FCS; Gibco Life Technologies) and 1\% penicillin-streptomycin. Cells were cultured on collagen-coated 24-well plates (BD Europe) at $5 \times 10^{6}$ cells/well. Single colonies of OECs, appearing with endothelial morphology after 3-4 weeks in culture, were trypsinized and expanded during several passages in a splitting ratio of $1: 2$ or $1: 3$. OECs from two donors were used in this study. The endothelial phenotype of these cells was stable in long-term culture, assessed previously using RT-PCR, immunofluorescence, flow cytometry and angiogenesis assays.

\subsection{Isolation of human osteoblasts}

Bone fragments were derived from consenting patients undergoing orthopaedic or trauma surgery procedures. This was approved by the responsible ethical committee. Primary osteoblasts were isolated and cultured according to an outgrowth protocol, as previously described (Fuchs et al., 2009c; Hofmann et al., 2003). In brief, bone fragments were minced and rinsed several time in phosphate-buffered saline (PBS), after which the bone tissue was digested by collagenase (Type IV C-5138, Sigma, Deisenhofen, Germany) for $1 \mathrm{~h}$ at $37^{\circ} \mathrm{C}$. After this digestion step, the bone fragments were again rinsed several times with PBS. The bone fragments were incubated in DMEM/Hams F12 (Gibco) supplemented with $20 \%$ FCS and $1 \%$ penicillin-streptomycin to foster outgrowth of the cells from the bone fragments. Confluent cultures were passaged $1: 3$ using acutase (PAA) and further cultured in DMEM/Hams F12 including 10\% FCS.

\subsection{Generation of SPCL co-culture constructs}

SPCL scaffolds were generated and sterilized as previously described (Gomes et al., 2003). Prior to cell seeding, the scaffolds were coated with fibronectin according to standard protocols (Santos et al., 2007). SPCL was seeded with 250000 primary osteoblasts and 250000 OECs for the co-cultures. The same cell culture medium, EGM-2 with 5\% FCS, was used for co-cultures according to a previously established protocol, without additional supplementation with angiogenic growth factors (Fuchs et al., 2007). The cells were cultured in vitro for a total of 7 days prior to implantation. OECs from each of the two donors individually were used to generate constructs.

\subsection{In vivo assessment of SPCL co-cultures}

The Committee on the Use of Live Animals in Teaching and Research, Rheinland-Pfalz, Germany, approved this animal protocol. Twenty 6 week-old male SCID mice (Charles River Laboratories) were housed under 
standard conditions and provided with water ad libitum, artificial light and normal mouse pellets (Laboratory Rodent Chow, Altromin) at the Laboratory Animal Unit, Johannes Gutenberg University, Mainz, Germany. The in vitro-generated SPCL constructs were embedded in $400 \mu \mathrm{l}$ Matrigel ${ }^{\circledR}$ before implantation. After gelation the constructs were implanted in preformed subcutaneous pockets of the subscapular region, as described previously (Fuchs et al., 2009c; Ghanaati et al., 2010). Briefly, the shaved skin of the rostral portion of the interscapular region was incised and the biomaterial was placed into a subcutaneous pocket under the thin skin muscle of the animal. The wound was subsequently stitched with 6.0 Prolene (Ethicon). Each surgical procedure was carried out under strictly aseptic conditions and care was taken to close the operation site immediately after biomaterial implantation, with incisions being accurately stitched. All animals survived the operation procedure and in all cases the implantation site healed without complications. In total, 10 animals received SPCL co-cultures with OECs from donor 1 and 10 animals received scaffolds with OECs from donor 2. Additionally, two constructs from each OEC donor were processed for histology at the time of implantation (time zero controls). Two animals from each donor subgroup were sacrificed with an overdose of ketamine and xylazine at days 2, 5, 8, 11 and 14 following implantation, in order to conduct a dynamic study on the transformation of the cell constructs in vivo.

\subsection{Histological preparation}

Immediately after death, the implants of all groups were explanted together with the surrounding peri-implant tissue and fixed in $4 \%$ formalin for $24 \mathrm{~h}$ for further histological and immunohistochemical analysis. The implant site of each animal was cut into three segments to include the SPCL constructs. After fixation, the segments were dehydrated in a series of alcohols, transferred to xylene and embedded in paraffin. Serial 3-4 $\mu \mathrm{m}$ thick paraffin sections were prepared from the central section, deparaffinized and rehydrated. Endogenous peroxidase activity was quenched by treatment with $3 \% \mathrm{H}_{2} \mathrm{O}_{2}$ in demineralized water. After blocking with serum-free Protein Block (Dako), the sections were stained with haematoxylin and eosin (H\&E) and immunohistologically stained for specific endothelial and osteogenic markers. To identify OECs, one section was stained with a mouse antibody against human-specific von Willebrand factor (vWF) or CD 31 (PECAM) (Dako). To identify all humanderived cells within the implantation bed, a section was stained with a rabbit antibody to vimentin (Dako). The use of serial sections of CD 31 and vimentin enabled the detection of osteoblasts within the implantation bed, since these cells were vimentin-positive and CD 31negative. OECs stained positive for both markers. After incubation with primary antibodies, the Dako REAL ${ }^{\mathrm{TM}}$ En Vision ${ }^{\mathrm{TM}}$ Detection System, Peroxidase/DAB ${ }^{+}$, mouseanti-rabbit (Dako) was used, which visualizes the reaction with diaminobenzidine. As negative control for each of the above stainings performed, one section was treated similarly without adding any primary antibody. All control stains were negative. Isolectin B4 (Vector Laboratories) was used to stain murine endothelial cells, which were then visualized with diaminobenzidine.

OEC-derived vessels were detectable by staining for human-specific von Willebrand factor as well as humanspecific CD31. For the results shown here, only CD31 is shown. Murine vessels were detectable by isolectin B4 and were distinguishable from human-specific OEC-derived vessels. These isolectin-stained sections were used for quantification of host-mediated scaffold vascularization, but no murine-specific staining is shown in the figures. Previous reports have demonstrated the detection of OECderived and murine-derived vessels at day 14 after implantation using human-specific vWF and isolectin B4, respectively (Fuch et al., 2009c). This report focuses primarily on the OEC-derived microvessels and exemplifies this using CD31 staining, although vWF and isolectin stainings were also performed for verification and quantification.

\subsection{Histomorphometric analysis of vascularization}

Histomorphometric analysis was performed using the software NIS-Elements (Nikon) according to the manufacturer's instructions and following a protocol that we had previously established (Ghanaati et al., 2009). Briefly, images were obtained with a DS-Fi1/digital camera connected to an Eclipse 80i histological microscope (Nikon), which was equipped with an automatic scanning table (Prior). A total scan, one large image assembled from 100-120 images of the region of interest containing the biomaterial and the corresponding peri-implant tissue was taken by using a $\times 100$ magnification at a resolution of $2500 \times 1200$ pixels. For each animal, two consecutive slides that were stained with vWF or CD31 antibody were used to detect the OEC-derived vessels and an additional two sections stained with isolectin B4 were used to detect murine-derived vasculature. Using the NIS-Elements 'annotations and measurements' tool, the total biomaterial area was measured and humanderived (OEC) and murine-derived (host) vessels were marked separately. Human cell-lined capillaries containing intraluminal erythrocytes were quantified as vessels having undergone anastomosis and considered to be functional vasculature in vivo. The total number of each type of vessel was quantified for each slide. The planar density of specific vessels was determined from the total number of each type of vessel divided by the total biomaterial area in $\mathrm{mm}^{2}$, providing a measure of density in terms of vessels $/ \mathrm{mm}^{2}$. The total scaffold vascularization (humanand murine-derived) was determined by summing the totals for each of the specific cell types. For each time point, a mean number of vessels $/ \mathrm{mm}^{2}$ was determined for each donor, with $n=2$ animals per time point per donor. 

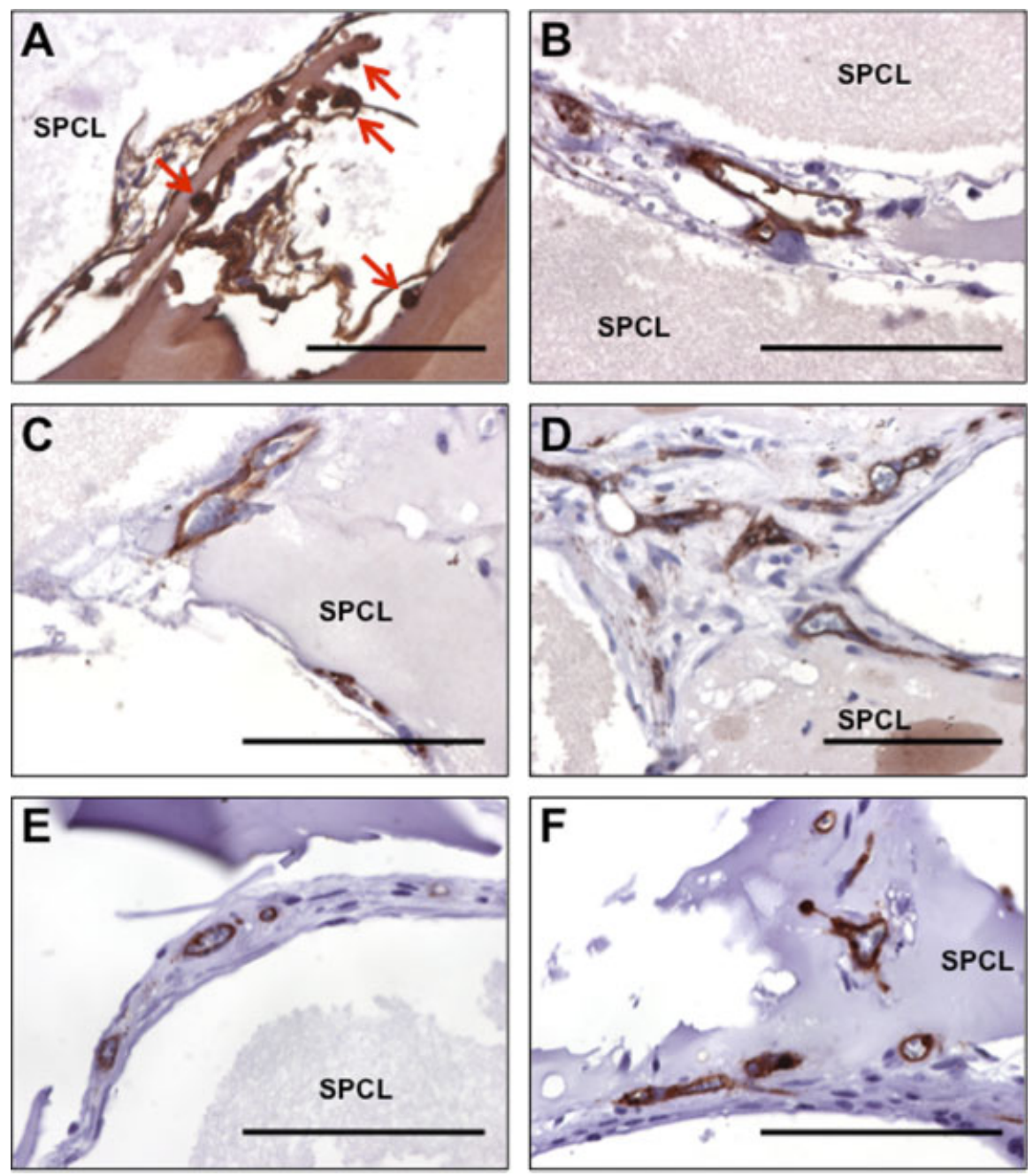

Figure 1. Immunohistochemical staining for human endothelial marker CD31 in OEC-pOB co-cultures at the time of implantation (time zero control) (A), with arrows indicating sprout-like structures. Implantation time points of 2 (B), 5 (C), 8 (D), 11 (E) and 14 (F) days are also shown. At all post-implantation time points there was evidence of perfused vascular structures. Magnification $=\times 400(\mathrm{~A}, \mathrm{D})$ and $\times 600(\mathrm{~B}, \mathrm{C}, \mathrm{E}, \mathrm{F})$; all scale bars $=100 \mu \mathrm{m}$

\section{Results}

Human-specific immunohistochemical detection of OECs following 7 days of in vitro co-culture with $\mathrm{pOB}$ and immediately prior to implantation (time zero control) demonstrated the presence of a connective tissue-like formation between the fibres of the SPCL mesh (Figure 1A). The CD31-positive staining of this preimplantation sample revealed the formation of sproutand vessel-like structures on the surface of the SPCL (Figure 1A, arrows). After 2 days of implantation in vivo, there was already some evidence of human-specific vessel structures within the mesh of the material bed in close proximity to the murine vessels (Figure 1B). These vessels were already anastomosed with the host vascular network at day 2 , as indicated by visible erythrocytes within the lumen of these human-specific vessels (Figure 1B). During days 2-14 the number of OEC-derived microvessels increased, as did the number that showed evidence of perfusion with murine erythrocytes (Figure 1C-F). At the end of the observational period, following 14 days of implantation, the SPCL constructs were homogenously vascularized by both OEC- and murine-derived vessels.

Histomorphometric analysis of the density of OECderived human vessels within the implantation bed was performed from a total scan of a histological slide of the implantation bed that was stained with an antibody to human-specific CD31 (Figure 2). In total, $n=2$ animals/donor/time point were used. At day 2 after implantation, there were already $2.7( \pm 0.6)$ and $4.0( \pm 0.7)$ OEC-derived vessels $/ \mathrm{mm}^{2}$ for donors 1 and 2 , respectively. This number continued to increase, and by day 14 there were $11.4( \pm 1.8)$ and $17.8( \pm 0.8)$ OEC-derived vessels $/ \mathrm{mm}^{2}$, for OECs from each of the two donors. Regardless of donor, the trend was for an increase in OEC-derived vascular structures over the implantation time. Quantification of vascularization arising from murine endothelial cells demonstrated similar trends (Figure 2). By day 14 of implantation, there were a total of $13.8( \pm 3.8)$ and $11.9( \pm 1.9)$ murine vessels $/ \mathrm{mm}^{2}$ for constructs from each of the two OEC donors, respectively. The rate of vascularization 

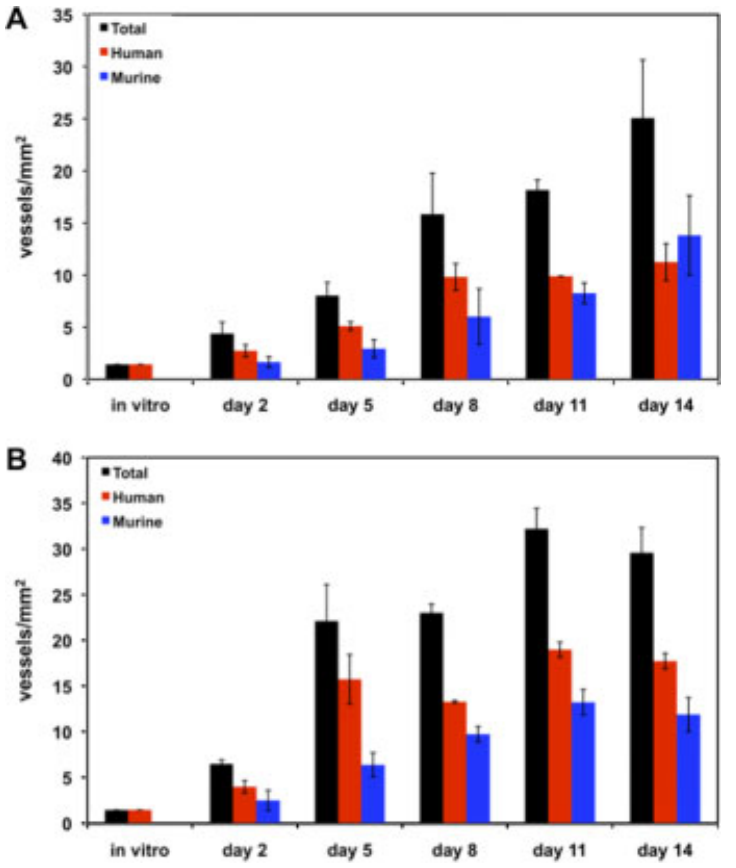

Figure 2. Histomorphometric quantification of total blood vessels (black), the sum of which derives from human OECs (red) and murine endothelial cells (blue) for donor 1 (A) and donor 2 (B). Vessel number is expressed per scaffold area at various times post-implantation and $n=2$ per time point per donor

and number of vascular structures was similar for both human- and murine-derived vessels. By the end of the study, vascularization arising from OECs accounted for $44.9 \%$ and $60.0 \%$ of total scaffold vascularization for donors 1 and 2, respectively. This indicates a substantial functional contribution of the precultured OECs towards overall scaffold vascularization.

Histological results from the various in vivo time points showed no sign of bone-specific matrix formation, either on the surface of the SPCL material or within the implantation bed. However, immunohistochemical detection of osteoblasts by means of serial vimentinand $C D$ 31-stained sections demonstrated that the pOB cells from the co-culture remained present and viable throughout the study, and were embedded in an extracellular matrix (Figure 3A, B). Moreover, staining consecutive sections (Figure 3A, C and B, D) with vimentin (Figure 3A, B) and CD 31 (Figure 3C, D) revealed co-localization of pOBs and OECs both at the scaffold surface and within the SPCL fibres. These pOBs surrounded the OEC-lined vessel, serving what appeared to be a type of pericyte-like function in supporting these OEC-derived vessels.

\section{Discussion}

The potential of human outgrowth endothelial cells to contribute to vascularization in complex tissueengineering strategies has been established by our group and others (Fuchs et al., 2006a, 2009a). Additionally, we have demonstrated potential for mature endothelial cells derived from human dermal tissue to act in a similar capacity (Unger et al., 2004, 2005, 2007). Observations with both OECs and other primary endothelial cells, originally demonstrated in vitro, have also been verified in vivo (Fuchs et al., 2009c; Unger et al., 2010). In one such study, we found co-cultures of OEC and pOB on SPCL scaffolds to have a high potency for the formation of human-specific vascular structures when transplanted in vivo (Fuchs et al., 2009c). This study, which focused on a single histological endpoint, indicated both the formation of OEC-derived vessels and a contribution to this phenomenon from the osteoblast component, acting as a natural source for potent angiogenic signalling within the co-culture. However, further mechanisms regarding the time course of the OEC-derived vessel formation in vivo and the further potential roles of osteoblasts were not completely elucidated. In the present study, we have examined the formation of these OEC-derived vessels in a more dynamic approach, with several histological time points and total implant histomorphometric analysis at each time point, in order to determine the time course of OEC-derived vessel formation along with the dynamics of total scaffold vascularization by both OEC- and murinederived vessels. Additionally, we have used vimentin human-specific staining, which was not performed in our previous study, coupled with CD 31 staining to specifically detect pOBs within the co-culture, in order to determine the specific location and potential contribution of these cells to OEC-derived vessel formation.

In this investigation, along with previous studies by our group (Fuchs et al., 2007), it is apparent that even prior to implantation, the OEC component was primed for the formation of vascular structures. The bud- or sprout-like structures seen in the histology of these scaffolds, following in vitro co-culture but prior to implantation, are reminiscent of the first stages of angiogenesis, where endothelial cells begin to reorganize and form sprouts that eventually lead to new vessels (Carmeliet, 2005; Folkman, 2003). Following 2 days of implantation, there was evidence of OEC-specific vessels with apparent lumena. The presence of erythrocytes within these lumena indicates that some human-derived vascular structures were already anastomosed to the host blood supply and were functionally contributing to vascularization of the implantation bed after only $48 \mathrm{~h}$ in vivo. These findings indicate the potential of OECs to form microvessel-like structures during in vitro cultivation that enable rapid anastomoses upon implantation, suggesting this as a potential 'natural' anastomosis technique for tissue engineering. Histomorphometric quantification of human-specific vessels demonstrated that the number of perfused human-derived vascular structures increased approximately four-fold from day 2 to day 14 of implantation. Although there appeared to be some differences in cell potency between the two OEC donor sources, both donors demonstrated an increase in human-derived vascularization of the scaffold over the evaluation period. One of the main differences between 
Human Vimentin
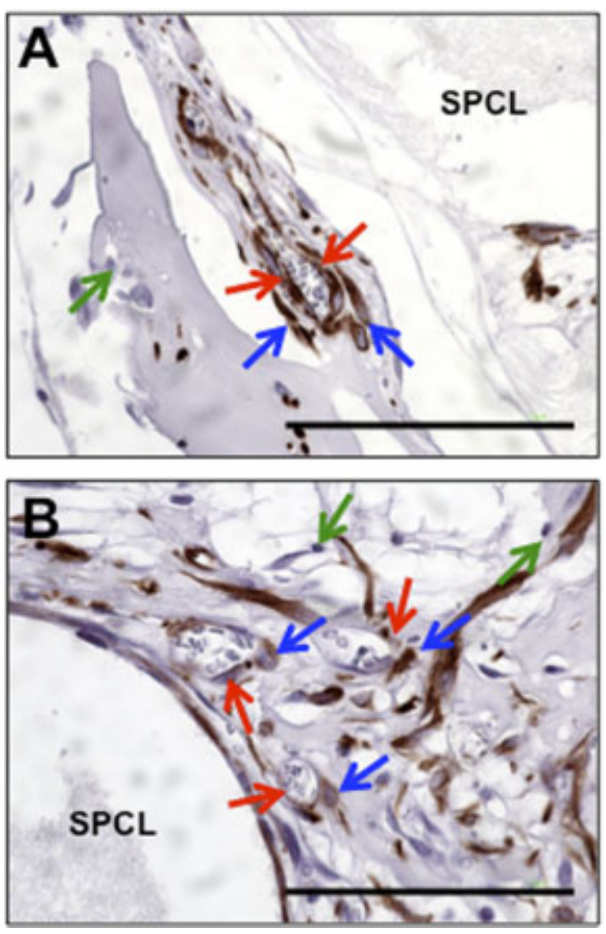

Human CD31
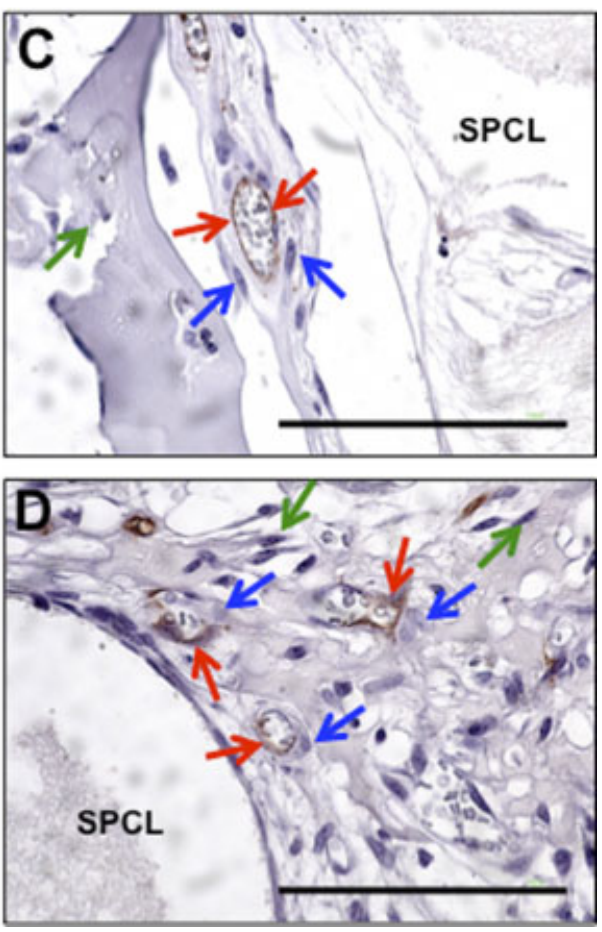

Figure 3. Immunohistochemical staining for vimentin (left), which specifically stains human cells within the implantation bed (both osteoblasts and OEC-derived endothelial cells), along with staining of the serial histological sections with an antibody specific to human CD31 (right) to visualize only OEC-derived endothelial cells. Serial sections (A, C) and (B, D) indicate the co-localization of pOBs and OECs, with the pOBs immediately surrounding the OEC vessel structures. Red arrows, OEC-derived endothelial cells; blue arrows, human osteoblasts; green arrows, murine-derived cells, which are negative for both stains. Magnification $=\times 600$ for all images; scale bars $=100 \mu \mathrm{m}$

the two donors (Figure 2) is that in one the principal acceleration of vessel growth took place between days 2 and 5 , whereas in the other donor this took place later, that is, between days 5 and 8. Moreover, the OECderived vessels made a substantial contribution to overall scaffold vascularization, as the number of vessels arising from OECs was similar to the number arising from the host. This is further evidence of the potency of this cell population to contribute to scaffold vascularization. The present study demonstrates the potential of preseeded OECs in cell-based tissue engineering strategies as a supplemental vascularization source, especially when host-mediated vascularization proves insufficient by itself, to fully integrate a biomaterial within the implantation bed in an appropriate time frame and with a sufficient vascular density.

Another finding in this study could help elucidate the contribution of co-cultured osteoblasts to OEC-derived vessel formation. Osteoblasts are known to produce a number of signals, including paracrine factors such as VEGF and structural components such as collagen I, which exert a pro-angiogenic effect on the cocultured endothelial cells and enhance the formation of vessel-like structures (Fuchs et al., 2009c; Unger et al., 2007). Our histological evidence from serial sections demonstrates co-localization of osteoblasts and OECs, with the osteoblasts forming a surrounding layer on the OEC-derived microvessels. These findings suggest that the contribution of osteoblasts goes beyond only a paracrine effect, and that the cell body of the osteoblasts may serve a structural role in the formation of these new vessels. Thus, osteoblasts may act as pericyte-like cells within these co-cultures by contributing structural and paracrine support to stabilize the endothelial-derived vascular structures. Such a role may be similar to that of an adventitial layer in normal vessel physiology, where mural cells, including fibroblasts, smooth muscle cells and other pericytes, provide structural support to vessels and enhance angiogenesis (Gerhardt and Betscholtz, 2003; Shepro and Morel, 1993). A connection between osteoblasts and pericytes is not without precedent. However, in this respect most literature findings indicate the potential of pericytes as pluripotent progenitors that can undergo osteogenic differentiation (Brighton et al., 1992; Doherty et al., 1998; Reilly et al., 1998). To our knowledge, there is not a significant body of literature that suggests the potential of osteoblasts to act in a pericytelike role. However, given the demonstrated similarities in phenotypic expression (Reilly et al., 1998), perhaps osteoblasts do have some capability to serve in this pericyte-like role. This phenomenon may arise specifically in co-cultures, where no other cell population exists to support the endothelial cells in the vessel formation process. Therefore, in co-cultures osteoblasts might be committed to this role as the default cell source for cell-based structural support of vessels. Nevertheless, the 
findings of this study, along with previous work, point to a possible synergy between the pOBs and OECs, in which pOBs may be able to provide both the chemical cues and structural support needed for OEC to rapidly form vessels.

The finding that OECs rapidly vascularize SPCL scaffolds in co-culture with $\mathrm{pOB}$ after only 2 days of implantation in vivo is promising for complex tissue engineering strategies, in which a rapid scaffold vascularization is desired. The rapid formation of anastomoses with the host vasculature after only $48 \mathrm{~h}$ is quite exciting and points to the potency of this cell source. The number of OEC-derived vessels increased over the implantation time with a density similar that of host-derived vessels. Furthermore, evidence is also provided for the multifunctional role of pOBs in the co-culture, as a structural pericyte-like support for the OEC-derived vessels was observed histologically. These findings, together with previous in vitro and in vivo studies by our group, demonstrate the potential of cellular crosstalk in co-cultures of endothelial cells and osteoblasts to provide an effective cell-based approach for tissue engineering.

\section{Conclusion}

The goal of our study was to establish the time scale over which human-specific vessels arise from cocultures of osteoblasts and endothelial cells in vivo. Additionally, this study further focused on the potential roles osteoblasts may play in the formation of OEC-derived microvessels. Our findings demonstrated that humanspecific vascular structures begin to form during in vitro precultivation and that these structures anastomosed with host vasculature within $48 \mathrm{~h}$ of implantation, as there was evidence of human-derived vessels visibly perfused with erythrocytes at this early time point. The number of human cell-derived vessels increased with time, irrespective of donor source, although the source seemed to result in different cell potency. An additional exciting finding was made histologically. While a proangiogenic paracrine contribution from osteoblasts has been previously established, we have demonstrated here the potential of these cells to serve additionally as structural vessel wall components, similar to that of pericytes in normal vascular physiology. The findings of this study further demonstrate the synergistic and multifunctional role of osteoblasts in co-culture with endothelial cells as an exciting strategy for cell-based complex tissue engineering.

\section{Acknowledgements}

The authors would like to thank Mrs B Pavic and Mrs U. Hilbig for their excellent technical assistance. This work was financially supported by grants from the European Commission (EXPERTISSUES Contract No. 500283-2) and the German Federal Ministry of Education and Research, BMBF (German-Chinese Cooperation in Regenerative Medicine; Contract No. 0315033).

\section{References}

Au P, Daheron LM, Duda DG, et al. 2008; Differential in vivo potential of endothelia progenitor cells from human umbilical cord blood and adult peripheral blood to form functional long-lasting vessels. Blood 111(3): 1302-1305.

Brighton CT, Lorich DG, Kupcha R, et al. 1992; The pericyte as a possible osteoblast progenitor cell. Clin Orthop Relat Res 275: 287-299.

Carmeliet P. 2005; Angiogenesis in life, disease and medicine. Nature 438(7070): 932-936.

Doherty MJ, Ashton BA, Walsh S, et al. 1998; Vascular pericytes express osteogenic potential in vitro and in vivo. $J$ Bone Miner Res 13(5): 828-838.

Folkman J. 2003; Fundamental concepts of the angiogenic process. Curr Mol Med 3(7): 643-651.

Fuchs S, Dohle E, Kolbe M, et al. 2009a; Outgrowth endothelial cells: sources, characteristics and potential applications in tissue engineering and regenerative medicine. Adv Biochem Eng Biotechnol (in press) [DOI: 10.1007/10_2009_65].

Fuchs S, Ghanaati S, Orth C, et al. 2009c; Contribution of outgrowth endothelial cells from human peripheral blood on in vivo vascularization of bone tissue engineered constructs based on starch polycaprolactone scaffolds. Biomaterials 30(4): 526-534.
Fuchs S, Hermanns MI, Kirkpatrick CJ. 2006b; Retention of a differentiated endothelial phenotype by outgrowth endothelial cells isolated from human peripheral blood and expanded in longterm cultures. Cell Tissue Res 326(1): 79-92.

Fuchs S, Hofmann A, Kirkpatrick CJ. 2007; Microvessel-like structures from outgrowth endothelial cells from human peripheral blood in two-dimensional and three-dimensional co-cultures with osteoblastic lineage cells. Tissue Eng 13(10): 2577-2588.

Fuchs S, Jiang X, Schmidt H, et al. 2009b; Dynamic processes involved in the prevascularization of silk fibroin constructs for bone regeneration using outgrowth endothelial cells. Biomaterials 30(7): 1329-1338.

Fuchs S, Motta A, Migliaresi C, et al. 2006a Outgrowth endothelial cells isolated and expanded from human peripheral blood progenitor cells as a potential source of autologous cells for endothelialization of silk fibroin biomaterials. Biomaterials 27(31): 5399-5408.

Gerhardt H, Betsholtz C. 2003; Endothelial-pericyte interactions in angiogenesis. Cell Tissue Res 314(1): 15-23.

Ghanaati S, Orth C, Unger RE, et al. 2010; Fine-tuning scaffolds for tissue regeneration: effects of formic acid processing on tissue reaction to silk fibroin. $J$ Tissue Eng Regen Med (in press) [DOI: 10.1002/term.257].

Ghanaati S, Webber MJ, Unger RE, et al. 2009; Dynamic in vivo biocompatibility of angiogenic peptide amphiphile nanofibers. Biomaterials $\mathbf{3 0}(31)$ : 6202-6212.

Gomes ME, Sikavitsas VI, Behravesh E, et al. 2003; Effect of flow perfusion on the osteogenic differentiation of bone marrow stromal cells cultured on starch-based three-dimensional scaffolds. $J$ Biomed Mater Res A 67(1): 87-95.

Hofmann A, Konrad L, Gotzen L, et al. 2003; Bioengineered human bone tissue using autogenous osteoblasts cultured on different biomatrices. $J$ Biomed Mater Res A 67(1): 191-199.

Melero-Martin JM, Khan ZA, Picard A, et al. 2007; In vivo vasculogenic potential of human blood-derived endothelial progenitor cells. Blood 109(11): 4761-4768.

Reilly TM, Seldes R, Luchetti W, et al. 1998 ; Similarities in the phenotypic expression of pericytes and bone cells. Clin Orthop Relat Res 346: 95-103.).

Santos MI, Fuchs S, Gomes ME, et al. 2007; Response of micro- and macrovascular endothelial cells to starch-based fiber meshes for bone tissue engineering. Biomaterials 28(2): 240-248. 
Santos MI, Unger RE, Sousa RA, et al. 2009; Crosstalk between osteoblasts and endothelial cells co-cultured on a polycaprolactone-starch scaffold and the in vitro development of vascularization. Biomaterials 30(26): 4407-4415.

Shepro D, Morel NM. 1993; Pericyte physiology. FASEB $J$ 7(11): 1031-1038.

Silva EA, Kim ES, Kong HJ, et al. 2008; Material-based deployment enhances efficacy of endothelial progenitor cells. Proc Natl Acad Sci USA 105(38): 14347-14352.
Unger RE, Ghanaati S, Orth C, et al. 2010; The rapid anastomosis between prevascularized networks on silk fibroin scaffold generated in vitro with cocultures of human microvascular enothelial and osteoblast cells and the host vasculature. Biomaterials 31(27): 6959-6967.

Unger RE, Peters K, Huang Q, et al. 2005 Vascularization and gene regulation of human endothelial cells growing on porous polyethersulfone (PES) hollow fiber membranes. Biomaterials 26(17): 3461-3469.
Unger RE，Peters K, Wolf M, et al. 2004; Endothelialization of a non-woven silk fibroin net for use in tissue engineering: growth and gene regulation of human endothelial cells. Biomaterials 25(21): 5137-5146.

Unger RE, Sartoris A, Peters K, et al. 2007; Tissue-like self-assembly in co-cultures of endothelial cells and osteoblasts and the formation of microcapillary-like structures on three-dimensional porous biomaterials. Biomaterials 28(27): 3965-3976. 